\title{
The Validity of Two Neuromotor Assessments for Predicting Motor Performance at 12 Months in Preterm Infants
}

\author{
You Hong Song, $\mathrm{MD}^{1}$, Hyun Jung Chang, $\mathrm{MD}, \mathrm{PhD}^{1}$, Yong Beom Shin, $\mathrm{MD}, \mathrm{PhD}^{2}$, \\ Young Sook Park, MD, $\mathrm{PhD}^{1}$, Yun Hee Park, $\mathrm{MD}^{1}$, Eun Sol Cho, $\mathrm{MD}^{1}$
}

\begin{abstract}
${ }^{1}$ Department of Physical Medicine and Rehabilitation, Samsung Changwon Hospital, Sungkyunkwan University School of Medicine, Changwon; ${ }^{2}$ Department of Rehabilitation Medicine, Pusan National University Hospital, Pusan National University School of Medicine, Busan, Korea
\end{abstract}

\begin{abstract}
Objective To evaluate the validity of the Test of Infant Motor Performance (TIMP) and general movements (GMs) assessment for predicting Alberta Infant Motor Scale (AIMS) score at 12 months in preterm infants.

Methods A total of 44 preterm infants who underwent the GMs and TIMP at 1 month and 3 months of corrected age (CA) and whose motor performance was evaluated using AIMS at 12 months CA were included. GMs were judged as abnormal on basis of poor repertoire or cramped-synchronized movements at 1 month CA and abnormal or absent fidgety movement at 3 months CA. TIMP and AIMS scores were categorized as normal (average and low average and $>5$ th percentile, respectively) or abnormal (below average and far below average or $<5$ th percentile, respectively). Correlations between GMs and TIMP scores at 1 month and 3 months CA and the AIMS classification at 12 months CA were examined.

Results The TIMP score at 3 months CA and GMs at 1 month and 3 months CA were significantly correlated with the motor performance at 12 months CA. However, the TIMP score at 1 month CA did not correlate with the AIMS classification at 12 months CA. For infants with normal GMs at 3 months CA, the TIMP score at 3 months CA correlated significantly with the AIMS classification at 12 months CA.

Conclusion Our findings suggest that neuromotor assessment using GMs and TIMP could be useful to identify preterm infants who are likely to benefit from intervention.
\end{abstract}

Keywords Preterm infant, Neuromotor assessment, Prediction, Motor skills

Received May 24, 2017; Accepted August 23, 2017

Corresponding author: Hyun Jung Chang

Department of Physical Medicine and Rehabilitation, Samsung Changwon hospital, Sungkyunkwan University School of Medicine, 158 Paryong-ro, Masanhoewon-gu, Changwon 51353, Korea. Tel: +82-55-233-5450, Fax: +82-55-233-8366, E-mail: reh.chj@gmail.com

ORCID: You Hong Song (https://orcid.org/0000-0002-7521-6119); Hyun Jung Chang (https://orcid.org/0000-0001-9126-4316); Yong Beom Shin (https://orcid.org/0000-0001-5026-1696); Young Sook Park (https://orcid.org/0000-0003-2752-7120); Yun Hee Park (https://orcid.org/0000-00034707-2266); Eun Sol Cho (https://orcid.org/0000-0003-3439-7176).

(c) This is an open-access article distributed under the terms of the Creative Commons Attribution Non-Commercial License (http://creativecommons.org/ licenses/by-nc/4.0) which permits unrestricted noncommercial use, distribution, and reproduction in any medium, provided the original work is properly cited. Copyright @ 2018 by Korean Academy of Rehabilitation Medicine 


\section{INTRODUCTION}

An increase in the survival rate of preterm infants has led to a proportionate increase in the number of children with motor impairment [1]. As early infancy is a critical period of brain development, early identification of infants with motor dysfunction is important for the implementation of appropriate interventions for better developmental outcomes [2]. However, over diagnosis should be avoided as it causes unnecessary parental worry and medical expenses. The American Academy of Pediatrics reported quality-of-care indicators for the neurodevelopmental follow-up of preterm infants, and suggested that a multidimensional screening test should be performed, using standardized instruments, at least twice within 1 year after birth [3]. Rosenbaum [4] recommended that neonatal follow-ups should combine a standardized follow-up schedule (at the due date and at 3, 6, 12, 24, and 36 months) with interval evaluation of infants with perturbing developmental patterns.

Early neuromotor assessment, particularly in preterm infants, can be complicated because motor development during the first year of life is rapid and affected by biological, social, and environmental aspects $[5,6]$. An unstable rate of infant motor development may complicate the efforts put forward to evaluate the efficacy of early intervention programs [7]. Fluctuations in the percentile rank of repeated assessments may represent random variations in each session rather than actual changes in motor performance. In addition, preterm infants exhibit different gross motor trajectories compared with term infants [8]. These findings can be incorrectly labeled as abnormal [4]. Spittle et al. [6] systematically reviewed the clinimetric properties of neuromotor assessments for preterm infants and reported that the best predictive assessment tools are age-dependent. The general movements (GMs) assessment and Test of Infant Motor Performance (TIMP) are the most predictive tools before the age of 4 months, while the Alberta Infant Motor Scale (AIMS) is appropriate for older infants (8-12 months). Also, these three tools have been reported to exhibit highest levels of reliability.

TIMP is a discriminative and evaluative tool of motor performance and is used to evaluate infants under 4 months of age $[9,10]$. The tool evaluates the posture and movement control required for functional performance in early infancy [11]. At 3 months of corrected age (CA),
TIMP has been shown to identify $92 \%$ infants whose development will be delayed at 12 months of age [12] and $72 \%$ infants who will exhibit poor motor performance at preschool age (4 to 5 years) [13].

GMs are assessed by observing the spontaneous motor activity of an infant from the preterm period to 5 months CA [14]. This is a predictive and discriminative tool and has been reported to show great predictive accuracy for motor outcomes. In a systematic review of tests for the prediction of cerebral palsy (CP), the sensitivity and specificity of the GMs assessment were 98\% (95\% confidence interval [CI], 74\%-100\%) and 91\% (95\% CI, 83\%-95\%), respectively [15]. In other studies, GMs at 1 month exhibited excellent sensitivity, while those at 3 months exhibited great specificity for predicting motor dysfunction at 12 months $[16,17]$.

AIMS is a discriminative tool for measuring the gross motor development of infants aged 0 to 18 months [18]. In total, 58 items are assessed by observing infants in prone, supine, sitting, and standing positions. A previous study demonstrated correlation between AIMS scores and Bayley II scores at 12 months CA in preterm infants [19]. In a systematic review of neuromotor assessments for preterm infants, AIMS was found to be an appropriate discriminative and predictive tool at 12 months CA [6].

The objective of the present study was to evaluate the validity of TIMP and the GMs assessment at 1 month and 3 months CA for predicting the motor performance evaluated using AIMS at 12 months CA in preterm infants. We also determined the predictive value of TIMP for the motor outcomes at 12 months CA in infants with high and low risks of developing $\mathrm{CP}$ according to the GMs assessment.

\section{MATERIALS AND METHODS}

\section{Subjects and study design}

The medical records of preterm infants who were evaluated using AIMS at 12 months CA between April 2010 and April 2013 were retrospectively reviewed. Among them, those who visited the high-risk infant clinic at 1 month (between 2 and 5 weeks) CA and 3 months (between 12 and 13 weeks) CA were enrolled in this study. In our high-risk infant clinic, GMs and TIMP scores of preterm infants were evaluated once during the writhing period and once during the fidgety period. This study was approved by the Samsung Changwon Hospital Institutional Review Board (No. 2016-SCMC-020-00). 


\section{Procedure}

GMs are assessed by observing video recording of the infant in the supine position, in a calm and alert state with no external stimulation [14]. In the present study, GMs were evaluated at 1 month CA (writhing period) and 3 months CA (fidgety period). A single clinician with certified training in the GMs assessment analyzed each video. The inter-rater reliability between the two pediatric physiatrists, including the examiner in this study, was investigated for 203 preterm infants in 2009 (not published), and an excellent agreement was observed $(\gamma=0.972, p=0.001)$. Subjects for the present study were recruited from April 2010, after completion of the reliability study. The movements during the writhing period were classified as normal writhing movements, poor repertoire, cramped-synchronized movements, or chaotic movements. During the fidgety period, fidgety movements were considered present (continuous or intermittent), abnormal, or absent.

TIMP (version 5.1) comprises of 42 items, of which 13 assess spontaneous movements such as head centering, reaching, and individual finger movements and 29 assess the motor responses to various positions and the auditory and visual responses to stimulation [10]. Each item has its own scale, and the scores for each item are added to yield a total score. The total scores are categorized as average (within +1.0 and -0.5 standard deviation [SD] of the age mean), low average ( -0.5 to -1.0 SD below the age mean), below average ( -1.0 to $-2.0 \mathrm{SD}$ below the age mean), and far below average (below -2.0 SD below the age mean). TIMP was also administered at 1 month and 3 months CA by a pediatric physiatrist with experience with preterm infants.

At 12 months CA, AIMS was administered to observe spontaneous movements with minimal handling in developmental positions [18]. A total of 58 items were assessed by a pediatric physiatrist, with the infant in prone (21 items), supine (9 items), sitting (12 items), and standing (16 items) positions. Each item was scored as observed (1 point) or not observed (no point). All the scores were summed up to yield the total raw score, which was converted into an age-based percentile rank [20].

\section{Statistical analysis}

The categorical scores for the three tests were dichotomized as normal or abnormal using the following clas- sifications: GMs during the writhing period (normal; abnormal: poor repertoire, cramped-synchronized, or chaotic), GMs during the fidgety period (normal: present; abnormal: abnormal, or absent), and TIMP (normal: average and low average, abnormal: below average and far below average) $[21,22]$. For AIMS, the 5 th percentile was used as the cut-off value to categorize infants as normal or abnormal.

All statistical analyses were performed using R version 3.3.0 (Vienna, Austria; http://www.R-project.org/) and package 'epiR'. Correlations between GMs and TIMP scores at 1 month and 3 months CA and the AIMS classification at 12 months CA were analyzed using Fisher exact analysis. Receiver operating characteristic (ROC) curves were used to determine the sensitivity, specificity, positive, and negative predictive values and accuracy (with 95\% CIs) of TIMP and the GMs assessment at 1 and 3 months CA for predicting the AIMS classification at 12 months CA. Areas under the curve (AUC) were compared.

\section{RESULTS}

The study recruited a total of 44 preterm infants. The mean gestational age of the recruited infants was 31.2 \pm 3.1 weeks and the mean birth weight was $1,719 \pm 516 \mathrm{~g}$. The characteristics of the recruited infants are described in Table 1.

Table 1. Demographic characteristics of preterm infants included in the present study $(n=44)$

\begin{tabular}{lc}
\hline \multicolumn{1}{c}{ Characteristic } & Value \\
\hline Gestational age (wk) & $31.2 \pm 3.1(25-36)$ \\
Birth weight (g) & $1,719 \pm 516(820-3,040)$ \\
Gender & \\
$\quad$ Male & $20(45.5)$ \\
$\quad$ Female & $24(55.5)$ \\
Small for gestational age & $2(4.5)$ \\
\hline $\begin{array}{l}\text { Intraventricular hemorrhage } \\
\text { (grade III and IV) }\end{array}$ & $10(22.7)$ \\
Respiratory distress syndrome & $23(52.3)$ \\
Bronchopulmonary dysplasia & $8(18.2)$ \\
Patent ductus arteriosus ligation & $6(13.6)$ \\
\hline Necrotizing enterocolitis & $2(4.5)$ \\
\hline
\end{tabular}

Values are presented as mean \pm standard deviation (range) or number (\%). 


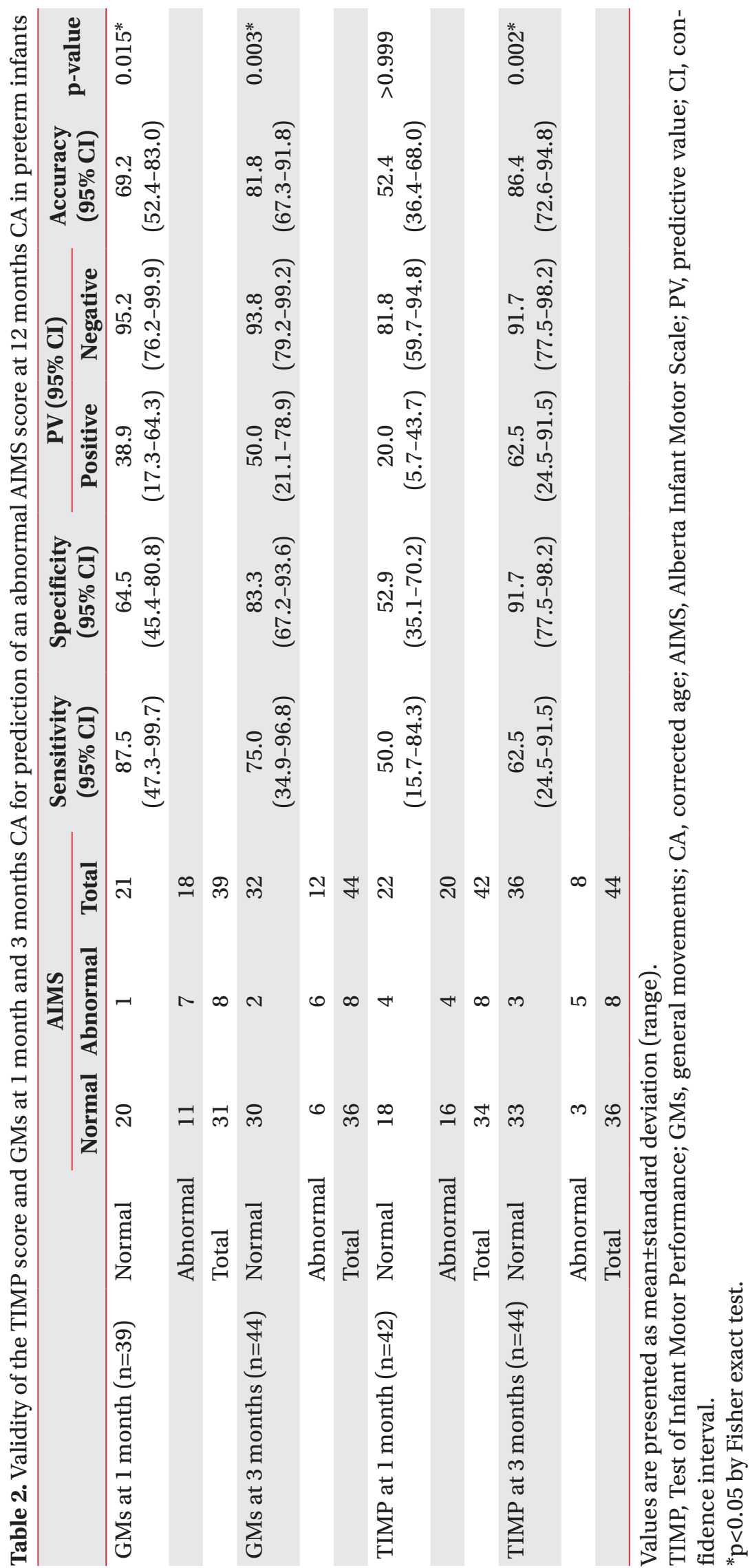




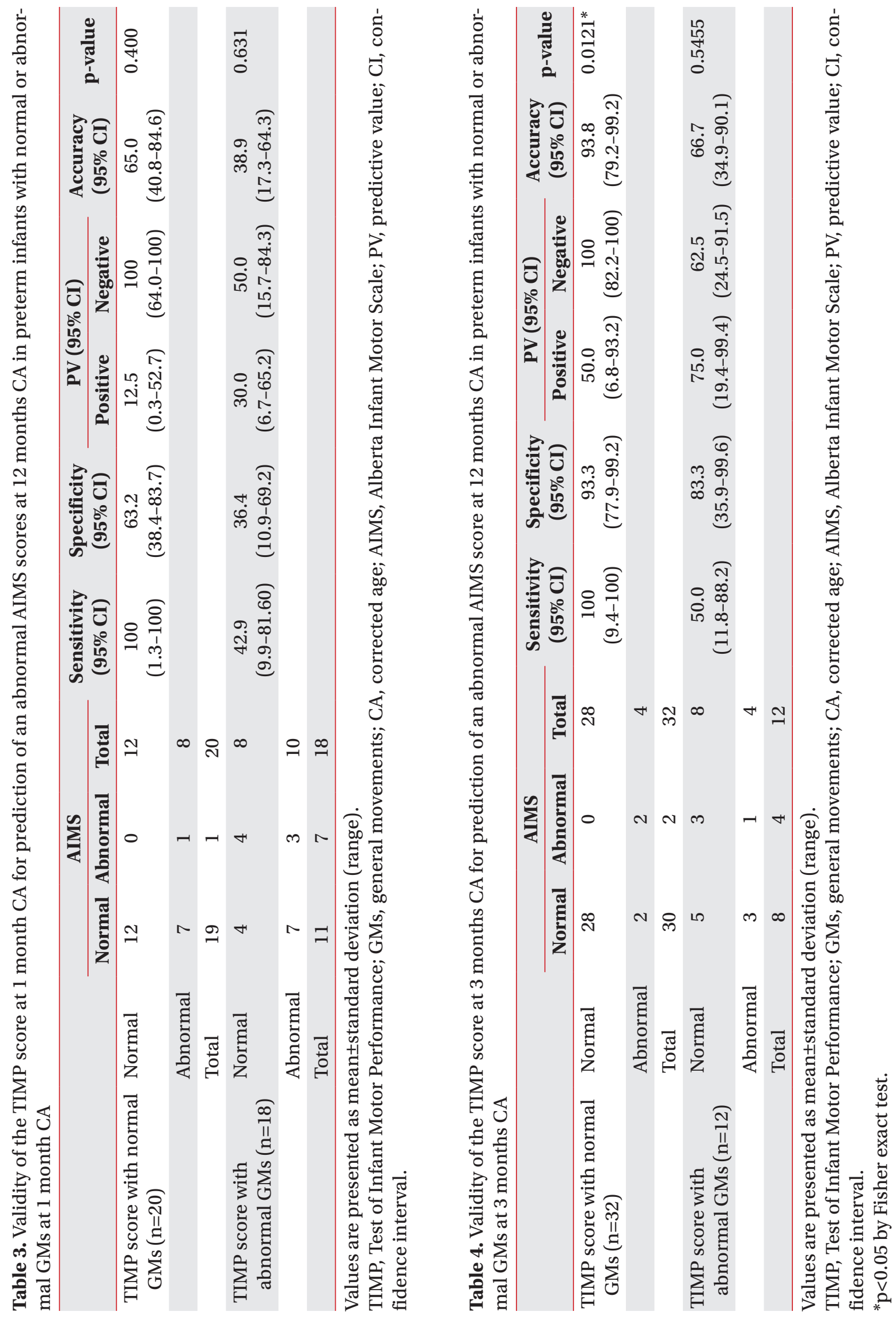


At 1 month CA, TIMP scores for two infants and GMs for five infants could not be evaluated as they were crying, irritable or asleep. Among 42 infants evaluated using TIMP at 1 month CA, 22 were categorized as normal and 20 as abnormal. Among 39 infants evaluated for GMs at 1 month CA, 21 showed normal GMs and 18 showed abnormal GMs. At 3 months, 36 infants exhibited a normal TIMP score and 8 exhibited an abnormal TIMP score, while 32 infants exhibited normal GMs and 12 exhibited abnormal GMs. According to AIMS, 36 infants were categorized as normal and 8 as abnormal at 12 months CA (Table 2).

\section{Correlations between GMs and TIMP scores and the AIMS classification}

GMs at both 1 month and 3 months CA showed a significant correlation with the AIMS classification at 12 months CA ( $p=0.015$ and $p=0.003$, respectively). The sensitivity, specificity, positive and negative predictive values along with accuracy (with $95 \%$ CIs) of the GMs assessment for the prediction of an abnormal AIMS score at 12 months are shown in Table 2.

There was a significant correlation between the TIMP score at 3 months CA and the AIMS classification at 12 months CA $(\mathrm{p}=0.002)$. However, the score at 1 month CA was not significantly correlated with the AIMS classification at 12 months CA ( $\mathrm{p}>0.999)$. The sensitivity, specificity, positive and negative predictive values, and accuracy (with 95\% CIs) of TIMP for the prediction of an abnormal AIMS score at 12 months are shown in Table 2.

\section{Correlation between TIMP scores and the AIMS classification according to GMs}

For infants with abnormal GMs at 1 month and 3 months CA, the TIMP score at both the time points did not show a significant correlation with the AIMS classification at 12 months CA (Tables 3, 4). Similarly, for infants with normal GMs at 1 month CA, the TIMP score at 1 month CA was not significantly correlated with the AIMS classification at 12 months CA (Table 3). However, a significant correlation between the TIMP score at 3 months CA and the AIMS classification at 12 months CA for infants with normal GMs at 3 months CA was observed ( $\mathrm{p}=0.012$ ) (Table 4). For infants with normal GMs at 1 month and 3 months CA, the sensitivity of the TIMP score at both the time points for the prediction of an abnormal AIMS score at 12 months CA was $100 \%$. All the infants with a normal TIMP score showed normal AIMS scores.

At 3 months CA, AUC and CI for GMs or TIMP alone were lower compared to the values of a combination of the two assessments, thus indicating that the overall outcome would be abnormal if any GMs and the TIMP score were abnormal. However, there was no statistical significance (Table 5).

\section{DISCUSSION}

In the present study, the TIMP scores at 3 months CA and GMs at 1 month and 3 months CA were significantly correlated with motor performance at 12 months CA in preterm infants. GMs at both 1 month (writhing period) and 3 months (fidgety period) CA were significantly correlated with the AIMS classification at 12 months CA. The GMs assessment is a valid and reliable assessment tool that predicts the likelihood of CP [23]. However, in agreement with our results, Spittle et al. [16] reported that the GMs assessment at 1 month CA exhibited an excellent sensitivity for the detection of motor dysfunction at 12 months CA, although with a high incidence of false-positive results, while that at 3 months CA exhibited higher specificity but a low sensitivity. Poor repertoire, categorized under abnormal writhing, may be followed by normal fidgety movements, although this may be re-

Table 5. Comparison of the predictability of TIMP and the GMs assessment (alone and in combination) at 3 months CA with regard to motor performance at 12 months CA in preterm infants $(n=44)$

\begin{tabular}{lcccc}
\hline & ROC & SE & 95\% CI & p-value \\
\hline TIMP at 3 months & 0.7917 & 0.0877 & $0.61981-0.96352$ & 0.086 \\
GMs at 3 months & 0.7708 & 0.0944 & $0.58576-0.95590$ & \\
Combined & 0.8889 & 0.0351 & $0.820002-0.95776$ & \\
\hline
\end{tabular}

TIMP, Test of Infant Motor Performance; GMs, general movements; CA, corrected age; ROC, receiver operating characteristic; SE, standard error; CI, confidence interval. 
lated to $\mathrm{CP}$, or mental or motor retardation. Furthermore, it has been reported to correlate more with milder brain abnormalities than with cramp-synchronized movement or absent fidgety movements $[14,24,25]$. Therefore, infants with poor repertoire are expected to exhibit normal motor performance at 12 months. This phenomenon can explain high sensitivity and low specificity of the GMs assessment at 1 month CA.

In the present study, the TIMP score at 3 months CA, and not at 1 month CA, was significantly correlated with the AIMS outcome at 12 months CA. Campbell et al. [12] reported that the 1-week TIMP score did not predict the 12-month AIMS percentile rank in multiple regression analysis. However, at later ages, TIMP scores were more accurate in predicting the 12-month AIMS percentile rank. They concluded that the TIMP score at 3 months of age has the highest validity for predicting motor performance at 12 months; this is consistent with our findings. When infants were evaluated at 1 month CA, the assessments were sometimes not easy because they were ill, irritable, or asleep thus leading to a lower score that did not represent their actual performance compared to normal AIMS score at 12 months CA. Furthermore, infants with increased extensor tone, who could later develop $\mathrm{CP}$, showed better performance in the assessment of neck extension, which comes across as a normal motor performance. Barbosa et al. [26] reported that there was no single best age for the identification of CP on the basis of scores for individual items of TIMP. However, they suggested that the maximum differences among groups were identified at 90 days of age. In another study, Barbosa et al. compared scores for TIMP items among children with CP, developmental delay, and typical development [11]. Children with CP showed better performance in the assessment of the extension pattern and developmental delay or regression in the assessment of antigravity movements and balanced flexion-extension pattern.

In the present study, the TIMP score at 3 months CA was predictive of motor performance at 12 months CA. However, a significant correlation was not observed for infants with abnormal GMs at 3 months. Spittle et al. [16] reported that GMs at 3 months are highly predictive of $\mathrm{CP}$ and significantly correlated with the AIMS classification at 12 months. However, when infants diagnosed with CP at a later stage were excluded from the analysis, the sensitivity of the GMs assessment at 3 months for predicting the 12-month motor outcome was lowered, exhibiting no significant correlation between GMs at 3 months CA and the AIMS score at 12 months CA. In the present study, for infants with normal GMs at 3 months CA, the TIMP score at 3 months CA was predictive of the AIMS outcome at 12 months CA. Among infants with normal GMs at 1 month CA, those with a normal TIMP score at 1 month CA showed normal AIMS scores at 12 months CA. Furthermore, one infant who showed normal GMs at 3 months CA and an abnormal AIMS score at 12 months CA showed an abnormal TIMP score at 1 month CA. However, there was no statistical significance. GMs may be useful for predicting $\mathrm{CP}$, but they have limited value for predicting other types of early motor dysfunctions. TIMP may be more accurate in predicting the AIMS score at 12 months in preterm infants with normal GMs (low risk of developing $\mathrm{CP}$ ) than in preterm infants with abnormal GMs (high risk of developing CP). We suggest that infants with normal GMs and normal TIMP scores can be followed-up after a long-term, while infants with abnormal GMs or abnormal TIMP scores require a close follow-up. Rosenbaum [4] reported that it is essential to include both qualitative and quantitative assessments in discrimination about early development.

This study has a number of limitations. First, the sample size was small. The power calculation for our sample size was conducted on the basis of findings from a previous study with a 0.05 significance level, and it was found that 44 subjects could provide a power of $87 \%$ for the detection of a correlation. Second, only one observer assessed all the patients; however, the observer was trained on performing Prechtls' method for the qualitative assessment of GMs and had exhibited excellent reliability. Third, we conducted rehabilitative interventions such as physical therapy for infants showing abnormal GMs or TIMP scores, which could have affected the outcomes. Fourth, the motor outcome was measured at 12 months $\mathrm{CA}$, and a longer follow-up study would have been beneficial. Finally, this study was a retrospective study; therefore, the results have limited validity. Further prospective or cohort studies are warranted.

In conclusion, we found that the TIMP score at 3 months CA and GMs at 1 and 3 months CA were correlated with motor performance assessed using AIMS at 12 months CA in preterm infants. In addition, we found that a normal TIMP score in preterm infants with a low risk of 
developing CP may be an accurate predictor of a normal AIMS outcome at 12 months. Our findings suggest that neuromotor assessments using both GMs and TIMP will be clinically helpful for predicting the motor outcome at 12 months in preterm infants and will be of aid in the identification of preterm infants who are likely to benefit from intervention.

\section{CONFLICT OF INTEREST}

No potential conflict of interest relevant to this article was reported.

\section{REFERENCES}

1. Bracewell M, Marlow N. Patterns of motor disability in very preterm children. Ment Retard Dev Disabil Res Rev 2002;8:241-8.

2. Vaccarino FM, Ment LR. Injury and repair in developing brain. Arch Dis Child Fetal Neonatal Ed 2004;89:F190-2.

3. Wang CJ, McGlynn EA, Brook RH, Leonard CH, Piecuch RE, Hsueh SI, et al. Quality-of-care indicators for the neurodevelopmental follow-up of very low birth weight children: results of an expert panel process. Pediatrics 2006;117:2080-92.

4. Rosenbaum P. Variation and "abnormality": recognizing the differences. J Pediatr 2006;149:593-4.

5. Johnson S, Marlow N. Developmental screen or developmental testing? Early Hum Dev 2006;82:173-83.

6. Spittle AJ, Doyle LW, Boyd RN. A systematic review of the clinimetric properties of neuromotor assessments for preterm infants during the first year of life. Dev Med Child Neurol 2008;50:254-66.

7. Darrah J, Redfern L, Maguire TO, Beaulne AP, Watt J. Intra-individual stability of rate of gross motor development in full-term infants. Early Hum Dev 1998;52: 169-79.

8. van Haastert IC, de Vries LS, Helders PJ, Jongmans MJ. Early gross motor development of preterm infants according to the Alberta Infant Motor Scale. J Pediatr 2006;149:617-22.

9. Campbell SK, Hedeker D. Validity of the Test of Infant Motor Performance for discriminating among infants with varying risk for poor motor outcome. J Pediatr 2001;139:546-51.
10. Campbell SK, Kolobe TH, Osten ET, Lenke M, Girola$\mathrm{mi}$ GL. Construct validity of the test of infant motor performance. Phys Ther 1995;75:585-96.

11. Barbosa VM, Campbell SK, Smith E, Berbaum M. Comparison of test of infant motor performance (TIMP) item responses among children with cerebral palsy, developmental delay, and typical development. Am J Occup Ther 2005;59:446-56.

12. Campbell SK, Kolobe TH, Wright BD, Linacre JM. Validity of the Test of Infant Motor Performance for prediction of 6-, 9- and 12-month scores on the Alberta Infant Motor Scale. Dev Med Child Neurol 2002;44: 263-72.

13. Kolobe TH, Bulanda M, Susman L. Predicting motor outcome at preschool age for infants tested at 7, 30, 60 , and 90 days after term age using the Test of Infant Motor Performance. Phys Ther 2004;84:1144-56.

14. Einspieler C, Prechtl H, Bos AF, Ferrari F, Cioni G. Prechtl's method on the qualitative assessment of general movements in preterm, term and young infants. London: Mac Keith Press; 2004.

15. Bosanquet M, Copeland L, Ware R, Boyd R. A systematic review of tests to predict cerebral palsy in young children. Dev Med Child Neurol 2013;55:418-26.

16. Spittle AJ, Boyd RN, Inder TE, Doyle LW. Predicting motor development in very preterm infants at 12 months' corrected age: the role of qualitative magnetic resonance imaging and general movements assessments. Pediatrics 2009;123:512-7.

17. Sustersic B, Sustar K, Paro-Panjan D. General movements of preterm infants in relation to their motor competence between 5 and 6 years. Eur J Paediatr Neurol 2012;16:724-9.

18. Piper MC, Darrah J. Motor assessment of the developing infant. Philadelphia: Saunders; 1994.

19. Jeng SF, Yau KI, Chen LC, Hsiao SF. Alberta infant motor scale: reliability and validity when used on preterm infants in Taiwan. Phys Ther 2000;80:168-78.

20. Majnemer A, Snider L. A comparison of developmental assessments of the newborn and young infant. Ment Retard Dev Disabil Res Rev 2005;11:68-73.

21. Snider L, Majnemer A, Mazer B, Campbell S, Bos AF. Prediction of motor and functional outcomes in infants born preterm assessed at term. Pediatr Phys Ther 2009;21:2-11.

22. Snider LM, Majnemer A, Mazer B, Campbell S, Bos AF. 
A comparison of the general movements assessment with traditional approaches to newborn and infant assessment: concurrent validity. Early Hum Dev 2008; 84:297-303.

23. Noble Y, Boyd R. Neonatal assessments for the preterm infant up to 4 months corrected age: a systematic review. Dev Med Child Neurol 2012;54:129-39.

24. Spittle AJ, Brown NC, Doyle LW, Boyd RN, Hunt RW, Bear M, et al. Quality of general movements is related to white matter pathology in very preterm infants. Pe- diatrics 2008;121:e1184-9.

25. Ferrari F, Cioni G, Prechtl HF. Qualitative changes of general movements in preterm infants with brain lesions. Early Hum Dev 1990;23:193-231.

26. Barbosa VM, Campbell SK, Sheftel D, Singh J, Beligere $\mathrm{N}$. Longitudinal performance of infants with cerebral palsy on the Test of Infant Motor Performance and on the Alberta Infant Motor Scale. Phys Occup Ther Pediatr 2003;23:7-29. 